OPEN ACCESS

Edited by:

Vasileios Papadopoulos, Aristotle University of Thessaloniki,

Greece

Reviewed by: Abhirup Banerjee,

Barts Health NHS Trust, United Kingdom

Thorvardur R. Halfdanarson,

Mayo Clinic, United States

*Correspondence:

Anna Sowa-Staszczak

anna.sowa-staszczak@uj.edu.pl

Specialty section:

This article was submitted to

Surgical Oncology,

a section of the journal

Frontiers in Oncology

Received: 30 March 2021 Accepted: 21 October 2021 Published: 12 November 2021

Citation:

Opalińska M, Sowa-Staszczak A

Grochowska A, Olearska H and Hubalewska-Dydejczyk A (2021) Value of Peptide Receptor Radionuclide Therapy as Neoadjuvant Treatment in the Management of Primary Inoperable Neuroendocrine Tumors.

Front. Oncol. 11:687925.

doi: 10.3389/fonc.2021.687925

\section{Value of Peptide Receptor Radionuclide Therapy as Neoadjuvant Treatment in the Management of Primary Inoperable Neuroendocrine Tumors}

\author{
Marta Opalińska ${ }^{1}$, Anna Sowa-Staszczak ${ }^{2 *}$, Anna Grochowska $^{3}$, Helena Olearska $^{2}$ \\ and Alicja Hubalewska-Dydejczyk ${ }^{2}$
}

${ }^{1}$ Nuclear Medicine Unit, Department of Endocrinology, Oncological Endocrinology and Nuclear Medicine, University Hospital, Kraków, Poland, ${ }^{2}$ Chair and Department of Endocrinology, Jagiellonian University Medical College, Kraków, Poland,

${ }^{3}$ Department of Radiology, University Hospital, Kraków, Poland

Introduction: Neuroendocrine neoplasms including neuroendocrine tumors (NETs) are often diagnosed as primary disseminated or inoperable. In those cases, systemic extensive therapy is necessary, but radical treatment is unlikely. As described in the literature, in some selected cases, peptide receptor radionuclide therapy (PRRT) may be used as a first-line/neoadjuvant therapy that allows further successful surgery. Such treatment may enable a reduction of total tumor burden or allow a radical treatment which improves the final outcomes.

Aim: This study aims to assess whether neoadjuvant PRRT could be a treatment option for patients with initially unresectable NETs.

Methods: Among the group of 114 patients treated with PRRT between the years 2005 and 2020, in 32 cases, it was the first-line therapy, mainly due to massive disease burden at the time of diagnosis. Among them, nine patients received PRRT as the first-line treatment due to the primary inoperable tumors with the intention of preoperative reduction of the tumor size in order to allow for a surgical treatment.

Results: Neoadjuvant PRRT enabled surgery in four out of nine (45\%) patients. Finally, in two out of four cases, the goal (radical surgery) has been achieved.

Conclusion: PRRT may be considered not only as a palliative but also as a neoadjuvant therapy in advanced, somatostatin-positive NETs that were initially inoperable.

Keywords: inoperable neuroendocrine tumors, PRRT, neoadjuvant therapy, NEN, NET (neuroendocrine tumors)

\section{INTRODUCTION}

Well-differentiated neuroendocrine tumors (NET G1, G2, and G3 according to WHO 2019 classification) are a widely heterogeneous group of malignancies regarding their place of origin, clinical presentation, hormone secretion, tumor growth, and metastases spread rate. A common feature of most NETs is overexpression of somatostatin receptors on their surface, which became the 
molecular basis for a theranostic approach using somatostatin analogs in the diagnosis and therapy of NETs.

However, in the presence of a localized, non-metastatic disease, surgery is the most effective treatment procedure which can enable complete recovery. In the case of advanced NETs not eligible for surgical treatment, several different antitumor therapeutic options may be used, but a chance for radical treatment is very low $(1,2)$. Among them, long-acting somatostatin analogs are the first-line treatment in the vast majority of NETs. Nevertheless, in some clinical settings, initial therapy with peptide receptor radionuclide therapy (PRRT) may bring benefits before further treatment. Several clinical trials proved PRRT to be one of the most effective therapeutic options in terms of objective responses in disseminated NET treatment. It has been demonstrated to be effective not only in improving progression-free survival (PFS) but also overall survival (OS) in those patients (3-5). Moreover, in selected cases, it may enable the resection of primarily inoperable tumors (6-8). For that reason, the rationale for the use of PRRT as first-line treatment may be especially valuable in case of extensive disease burden at the time of diagnosis, hormonal syndromes resistant to somatostatin analogs, or a chance for subsequent curative surgery. However, the overall outcome in the abovementioned clinical situations remains completely different.

The purpose of this study was to evaluate if PRRT used as neoadjuvant therapy in patients with NETs may enable radical surgery.

\section{MATERIALS AND METHODS}

Among a group of 114 patients treated in our center with PRRT between the years 2005 and 2020, 32 of them received PRRT as firstline therapy. Nine of them were qualified for PRRT with the intention of preoperative reduction of the tumor size, which could lead to potential subsequent radical surgery. The "unresectable primary tumor" was defined as extensive large vessel infiltration by neoplastic tissue or tumor invasion to adjacent organs, visualized on preoperative CT scans. All patients referred for preoperative PRRT were consulted by a multidisciplinary team including an oncological surgeon and a radiologist.

In this group, all patients had a histopathological diagnosis of well-differentiated NET according to the European Neuroendocrine Tumor Society-World Health Organization 2010 and 2017 grading system before PRRT, depending on the time of diagnosis. In eight patients, foregut tumors were present [in two in the lungs and in six in the pancreas (pNET)], and one patient was diagnosed with a midgut tumor (small intestine). In two patients, lesions were hormonally active (one insulinoma, one glucagonoma), and in another two, there was a suspicion of single liver metastasis detected in somatostatin receptor imaging (SRI) or computed tomography (CT) scans. Before and after PRRT, all of them were in generally good condition (Karnofsky index over 70\%).

All patients qualified for PRRT had a positive result (Krenning scores 3 and 4$)$ of SRI [ $(99 \mathrm{mTc})$ Tc-octreotide SPECT/CT or (68Ga) Ga-DOTA-TATE PET/CT]. Cytoreductive chemotherapy or longacting somatostatin analog was not used before PRRT in seven cases. Two patients received chemotherapy prior to PRRT with no response.
In all patients, 3 to 5 cycles of PRRT were applied. [90Y]YDOTA-TATE [mean cumulative dose $13.4 \mathrm{GBq}( \pm 1.44)$ ] and $[90 \mathrm{Y}] \mathrm{Y} /[177 \mathrm{Lu}] \mathrm{Lu}-\mathrm{DOTA}-\mathrm{TATE}$ (cumulative dose $14.8 \mathrm{GBq}$ ) were applied in eight patients and one patient, respectively. To reduce the radiation dose to the kidneys, as recommended, an infusion of amino acids (arginine and 2.5\% lysine) was administered.

The type of radiopharmaceutical used for PRRT depended on PRRT type availability in consecutive years. Routine blood count, liver function, and kidney function were assessed before each therapy cycle and at follow-up visits.

CT was performed 1-3 months prior to PRRT and 4-6 months after PRRT. Multidetector row spiral CT of $2 \mathrm{~mm}$ slice thickness and reconstruction increment were used after the administration of non-ionic contrast media. Further follow-up examinations were performed according to the applicable guidelines and the individual clinical course of the disease.

Diameter, volume, and the mean attenuation reduction of each lesion were calculated by CT image processing software.

Tumor response was assessed according to the Response Evaluation Criteria in Solid Tumors (RECIST) 1.1, where the partial response to the therapy is described as $\geq 30 \%$ decrease of the sum of the longest diameters of target lesions, whereas progression is a $\geq 20 \%$ increase of it (Table 1). The Choi criteria define objective response as a $\geq 10 \%$ decrease in the sum of tumor diameters or $\geq 15 \%$ decrease in the tumor density on contrast-enhanced CT scan (Table 1).

Response categories were assessed on a subsequent CT scan until the disease progression.

\section{Statistics}

Percentage changes in tumor diameter, volume, and density of tumor mass 1-3 months before and 4-6 months after PRRT were counted as well as the response to PRRT in RECIST 1.1 scale and Choi criteria. Additionally, the percentage of patients who underwent surgery (including complete surgical excision of the tumor) was assessed.

Finally, PFS and OS were calculated. PFS was defined as the time from the first PRRT to radiological or clinical disease progression or death from any cause.

\section{RESULTS}

The group of nine patients (six males and three females) were eligible to the analysis. The mean age of the patients equaled 53.78 years ( \pm 14.86 , range: $28-78$ years).

After the PRRT, the median tumor diameter changed by -1.6 $\mathrm{cm}$ (range from -3.7 to $0.3 \mathrm{~cm}$ ). The median tumor volume decreased by $105.0 \mathrm{~cm}^{3}$ (range from -186.2 to $34.7 \mathrm{~cm}^{3}$ ), whereas attenuation decreased by $9.1 \mathrm{HU}$ (range from -17.6 to $17.9 \mathrm{HU}$ ). There was no significant difference in the reduction of the tumor diameter, volume, and attenuation between pNET and other (not pNET) lesions (Table 2).

According to RECIST 1.1 criteria, stabilization of the disease (SD) and partial response (PR) were observed in six and one patient, respectively, and progressive disease (PD) was seen in two patients. In two patients, liver metastases described in the initial SRI were not 
TABLE 1 | Definition of radiological responses to therapy according to RECIST 1.1 and Choi criteria.

\begin{tabular}{|c|c|c|}
\hline & RECIST 1.0/1.1 & Choi \\
\hline Measurement & Largest diameter & Largest diameter + attenuation \\
\hline $\begin{array}{l}\text { Complete response } \\
\text { (CR) }\end{array}$ & Disappearance of all target lesions & Disappearance of all target lesions \\
\hline $\begin{array}{l}\text { Partial response } \\
\text { (PR) }\end{array}$ & $\begin{array}{l}\text { At least a } 30 \% \text { decrease in the sum of the greatest unidimensional diameters } \\
\text { of target lesions }\end{array}$ & $\begin{array}{l}\text { Decrease in tumor size } \geq 10 \% \text { or decrease in tumor density } \\
\geq 15 \% \text { on CT }\end{array}$ \\
\hline $\begin{array}{l}\text { Disease progression } \\
\text { (PD) }\end{array}$ & An increase of at least $20 \%$ in the sum of the diameters of target lesions & $\begin{array}{l}\text { Increase in tumor size } \geq 10 \% \text { and does not meet PR criteria by } \\
\text { tumor density }\end{array}$ \\
\hline $\begin{array}{l}\text { Disease stabilization } \\
\text { (SD) }\end{array}$ & Does not meet the criteria for $\mathrm{CR}$, $\mathrm{PR}$, or PD & \\
\hline
\end{tabular}

TABLE 2 | Changes in median (range) of diameter, volume, and attenuation of tumor before and after PRRT in pNET and not pNET patients.

\begin{tabular}{lcccc}
\hline & $\begin{array}{c}\text { Median difference of tumor diameter } \\
\text { before and after PRRT, cm (range) }\end{array}$ & $\begin{array}{c}\text { Median difference of tumor volume before } \\
\text { and after PRRT, } \mathbf{c m}^{\mathbf{3}} \text { (range) }\end{array}$ & $\begin{array}{c}\text { Median difference of tumor attenuation } \\
\text { before and after PRRT, HU (range) }\end{array}$ & $\begin{array}{c}\text { Statistical } \\
\text { significance }\end{array}$ \\
\hline pNET & $-0.4(-3.70$ to 0.30$)$ & $-7.8(-186.20$ to 34.72$)$ & $1.0(-17.60$ to 17.90$)$ \\
Not & $0.0(-1.47$ to 0.00$)$ & $-0.1(-125.94$ to -0.1$)$ & $-4.2(-4.40$ to 6.10$)$ \\
pNET & & & NS \\
\hline
\end{tabular}

pNET, pancreatic NET; not pNET, not pancreatic NET; NS, not significant.

found after PRRT on the follow-up SRI scans, but in one of them, a new SRI negative lesion was detected on CT examination.

According to the Choi criteria counted in eight patients, SD was observed in three, PR in three, and PD in two cases. The correspondence of those two scales was low and the evaluation of the PRRT results was comparable only in $50 \%$ of the cases (four patients) (Table 3 ).

The median time offollow-up was 56.9 months (range from 7.8 to 117.7 months). PRRT did not cause clinically important myelotoxicity or nephrotoxicity (CTCAE version 5.0 grades 3 and 4).

Among the whole group of patients, surgery was performed in four cases (45\%), but a radical procedure was possible only in two of them (22\%). The main cause of renouncement or ineffectiveness of surgery was an infiltration of the large vessels by neoplastic tissue, visualized on CT scans or found during the operation. No surgical complications which could be related to PRRT administration were observed. There was no perioperative mortality.

Two patients who underwent radical surgery are free from disease as of now, one of them for 27.13 months and another for 117.43 months. Both remain in the follow-up group. The assessment of radiological response to PRRT in patient no. 9 (treated radically) differed on the RECIST 1.1 and Choi scales, being SD and PR, respectively (Table 3).

In patient no. 8 , based on medical documentation, the tumor mass significantly decreased after PRRT, which then enabled surgical intervention. Unfortunately, the CT scan done after PRRT completion was not available.

Among other two patients who underwent incomplete surgery, PFS equaled 8.2 and 72.9 months.

In the group of patients who did not qualify for surgery, the median PFS was 21.5 months (range from 5.6 to 70.1 ). The median OS for the whole group was 56.9 months (range from 7.6 to 117.7) (Table 4). No significant difference in survival time was observed in patients stratified according to primary localization of NET (pNET vs. non-pNET).

\section{DISCUSSION}

Neoadjuvant therapy is an initial therapy which may be given to shrink the neoplastic tumor and enable further surgical intervention. It is widely used in different types of cancers including breast, pancreatic, and others, but not common in NETs due to usually large tumor burden at diagnosis.

According to current ENETS guidelines, various systemic therapies are available for locally advanced, metastatic, and progressive gastroenteropancreatic neuroendocrine tumors (GEP-NETs) (1). Long-acting somatostatin analog therapy is applied as a first-line treatment in the presence of somatostatin receptor (SSTR) expression at molecular imaging. The second- or third-line therapy regimens include chemotherapy with capecitabine and temozolomide (CAPTEM), PRRT, protein kinase inhibitors, streptozocin-based chemotherapy, or locoregional therapies, usually liver-directed $(2,3)$. PRRT is effective independently of the type of beta minus emitter (Y-90/ Lu-177) or somatostatin analog (TATE/TOC) being used $(9,10)$. Moreover, PRRT efficacy is high although the schemes of therapy and the use of specific radionuclide differ between centers. The direct effectiveness of PRRT in comparison with other types of therapy regimens is planned to be evaluated on the basis of ongoing or future clinical trials including comparison of PRRT to everolimus in progressing G1 and G2 GEP-NETs (COMPETE, ClinicalTrials.gov Identifier: NCT03049189) or to everolimus, FOLFOX, and CAPTEM in aggressive G2 and G3 GEP-NETs (COMPOSE, ClinicalTrials.gov Identifier: NCT04919226).

Among other treatment possibilities, temozolomide has shown antitumor activity in pNETs either as monotherapy or in combination with capecitabine (CAPTEM) or bevacizumab. The objective response rates ranged from 33\% (11) to 70\% (12), with the highest response rates in studies using CAPTEM. However, the use of CAPTEM regimen in patients with localized pNEN stratified by grade and neoadjuvant or 
TABLE 3 | Presentation and radiological and clinical outcomes of the patients.

\begin{tabular}{|c|c|c|c|c|c|c|c|c|c|c|c|c|c|c|}
\hline No. & Gender & $\begin{array}{l}\text { Place of } \\
\text { primary } \\
\text { tumor }\end{array}$ & $\begin{array}{l}\text { Metastases } \\
\text { to the liver }\end{array}$ & $\begin{array}{l}\text { Type } \\
\text { of } \\
\text { PRRT }\end{array}$ & $\begin{array}{l}\text { Change of } \\
\text { tumor diam- } \\
\text { eter after } \\
\text { PRRT }\end{array}$ & $\begin{array}{c}\% \text { change of } \\
\text { tumor volume } \\
\text { after PRRT }\end{array}$ & $\begin{array}{l}\text { Response to } \\
\text { PRRT in } \\
\text { RECIST cri- } \\
\text { teria }\end{array}$ & $\begin{array}{l}\% \text { change of } \\
\text { tumor attenua- } \\
\text { tion after PRRT }\end{array}$ & $\begin{array}{c}\text { Response } \\
\text { to PRRT in } \\
\text { CHOI } \\
\text { criteria }\end{array}$ & Surgery & $\begin{array}{l}\mathbf{R} \\
\mathbf{0}\end{array}$ & $\begin{array}{l}\text { Time to } \\
\text { progression } \\
\text { after PRRT }\end{array}$ & $\begin{array}{c}\text { Follow- } \\
\text { up } \\
\text { (months) }\end{array}$ & $\begin{array}{c}\text { Status } \\
\text { at last } \\
\text { follow- } \\
\text { up }\end{array}$ \\
\hline 1 & $\mathrm{~F}$ & Lung & No & $90 Y$ & $0 \%$ & $-0.6 \%$ & $\mathrm{SD}$ & $10.7 \%$ & $\mathrm{SD}$ & $\mathrm{N}$ & $\mathrm{N}$ & 62.5 & 62.5 & Dead \\
\hline 2 & M & Pancreas & No & $90 Y$ & $-48 \%$ & $-80.7 \%$ & $\begin{array}{l}\text { PD (new liver } \\
\text { lesion) }\end{array}$ & $3.5 \%$ & $\begin{array}{l}\text { PD (new } \\
\text { liver lesion) }\end{array}$ & Y & $N$ & 3.1 & 51.8 & Dead \\
\hline 3 & $\mathrm{~F}$ & Pancreas & No & $90 Y$ & $-1 \%$ & $12 \%$ & $\mathrm{SD}$ & $-25 \%$ & PR & $\mathrm{N}$ & $\mathrm{N}$ & 59.5 & 117.8 & Dead \\
\hline 4 & M & Pancreas & No & $90 Y$ & $5 \%$ & $5 \%$ & SD & $30 \%$ & $\mathrm{PD}$ & $\mathrm{N}$ & $N$ & 0.7 & 93.4 & Dead \\
\hline 5 & $M$ & $\begin{array}{l}\text { Small } \\
\text { intestine }\end{array}$ & No & $90 Y$ & $-21 \%$ & $-60 \%$ & $\mathrm{SD}$ & $-10 \%$ & PR & Y & $N$ & 65.0 & 105.6 & Dead \\
\hline 6 & M & Lung & No & $90 Y$ & $0 \%$ & $-3 \%$ & $\mathrm{SD}$ & $-9.0 \%$ & $\mathrm{SD}$ & $N$ & $N$ & 2.3 & 7.6 & Dead \\
\hline 7 & M & Pancreas & No & $90 Y$ & $-30 \%$ & $-83 \%$ & PR & $19 \%$ & $\mathrm{PD}$ & $\mathrm{N}$ & $N$ & 16.3 & 48.1 & Dead \\
\hline 8 & $\mathrm{~F}$ & Pancreas & Yes & $90 Y$ & $\mathrm{n} / \mathrm{a}$ & $\mathrm{n} / \mathrm{a}$ & PD & $\mathrm{n} / \mathrm{a}$ & $\mathrm{n} / \mathrm{a}$ & $\begin{array}{l}\text { Y (hemi- } \\
\text { hepatectomy) }\end{array}$ & Y & 3.8 & 116.2 & Alive \\
\hline 9 & M & Pancreas & Yes & $\begin{array}{l}177 \mathrm{Lu} / \\
90 \mathrm{Y}\end{array}$ & $-8 \%$ & $-18 \%$ & SD & $-11 \%$ & $\mathrm{PR}$ & Y & Y & 26.4 & 26.4 & Alive \\
\hline
\end{tabular}

$P D$, disease progression; $S D$, disease stabilization; $P R$, partial response; $R 0$, surgical resection assessed as radical in histopathology report; $Y$, yes; $N$, no; $n / a$, not available.

TABLE 4 | Long-term outcome of the patients who underwent PRRT as neoadjuvant therapy.

Features

Disease progression up to 6 months after PRRT

Time to progression, months; median (range)

Overall survival, months; median (range)

Surgeries after PRRT, $n$

Radical surgeries after PRRT, $n$

adjuvant therapy in comparison to somatostatin analog was associated with poorer OS (13), which raises doubts about the potential use of CAPTEM as first-line therapy even with the intention of using it as neoadjuvant therapy. In one of the studies, neoadjuvant CAPTEM regimen with or without radiation has been successfully applied in six pNETs with borderline resectable disease. All patients had radiological evidence of tumor regression after neoadjuvant treatment (two PR and four SD stabilization), and all of them could undergo successful resection of the primary tumor with negative margins in four out of six patients (14).

Throughout the 15 years of PRRT treatment in our center, we used both Y-90 and Lu-177 separately or as a tandem therapy combining Y-90 and Lu-177 with an activity ratio of 1:1. In all types of PRRT schemes, positive results were observed after the use of PRRT as first- or second-line therapy. In very few cases, PRRT was administered in an attempt to reduce the baseline tumor size with an intention of further radical surgical treatment. This approach offers hope for complete recovery which is not likely achievable with other forms of systemic treatment. Until now, there are only a few publications summarizing the use of PRRT as neoadjuvant therapy in NET patients, and a significant number of them relate to small groups of patients and case reports. The publication describing the largest group of patients who underwent neoadjuvant PRRT shows an encouraging rate of successful surgeries even in $31 \%$ of patients (9 out of 29 cases) (15). In our material, the rate of successful surgeries after PRRT was slightly lower (22\%) in comparison with the abovementioned publication, but the rate of complete recoveries still appears inspiring enough to consider such treatment in selected cases. It is worth emphasizing that among our patients, there was one case who presented single liver metastasis on CT/SRI scans prior to PRRT with no evidence of hepatic lesions on both CT and SRI scans after PRRT treatment. Similar results, including the cure of liver metastases by PRRT, were described in the past by a few authors (16-19). Those observations also encourage considering PRRT as neoadjuvant therapy even in the presence of a single liver metastases, especially if they are poorly available for locoregional treatment.

The second potential advantage of the use of PRRT at the beginning of treatment is a significant decrease of total tumor burden. This fact was clearly demonstrated mainly for $177 \mathrm{Lu}-$ DOTA-TATE therapy in one randomized trial (NETTER-1 trial) (3) and several non-randomized trials (20). According to a metaanalysis done on patients with disseminated pancreatic NETs, the pooled median PFS after PRRT was 25.7 months (95\% CI: 18.9-32.4 months) and was better than in patients treated with everolimus [PFS 14.7 months (95\% CI: 11.2-18.1 months)] (21), which is recommended as second-line treatment in disseminated pNETs. In our group, median PFS (in a corresponding group of patients who did not undergo surgery) was 21.5 months (range 5.6-70.1). The results obtained in our group are significantly better, which probably results from the selection of patients with a chance of radical surgery, i.e., with a relatively small disease burden, without multiple metastases. The PFS increase additionally encourages the use of PRRT at the beginning of treatment, especially if there is initially high tumor burden and when prolongation of PFS (less probable to achieve with the use of other systemic therapy) may be considered as an added benefit.

The results of PRRT assessed as disease regression, stabilization, or progression depend on the radiological method used for the evaluation of response to therapy. The most common methods used for that purpose are RECIST, Southwest Oncology Group (SWOG), or Choi criteria. RECIST scale (1.0 and more common nowadays 1.1) is already a radiological gold standard for the assessment of tumor response to cytoreductive treatment for different malignancies. However, this scale is hardly efficient in the validation of neoplasms with relatively slow growth (22). The main weakness of this scale is that it measures only the longest diameters of all selected target lesions, while the linear diameter does not vary sufficiently to correct estimation of total lesion 
volume. For this reason, scales such as the Choi criteria, which try to also take into account changes in the radiological density of lesions (reduction of attenuation, weaker contrast enhancement as the effect of neoplastic tissue necrosis), were created. Those scales are considered to be more useful in tumors with relatively slower growth. In our material, the response of seven out of nine patients (assessed as a PR or SD) to the treatment fits into one of those scales: seven out of nine in the RECIST scale and five out of eight into the Choi criteria. However, the same type of response in both scales was seen in four cases showing relatively poor compatibility (50\%) of both of those rating systems. Another side of the imperfection of those scales is seen in the example of a patient with a pancreatic NET producing insulin. After [90Y]Y/[177Lu] Lu-DOTA-TATE treatment, the tumor diameter decreased by $8 \%$, its volume by $18 \%$ and attenuation decreased by $11 \%$. It allowed us to assess the response to therapy as SD and PR according to RECIST and CHOI criteria respectively (Figures 1, 2). However, the reduction of tumor size and the decrease of tumor vascular involvement enabled curative surgery, confirming that neither of those radiological tools is highly effective in the preliminary assessment of PRRT efficacy nor does it predict a clinical outcome (patient was radically operated) (Figures 1, 2). It implies that it is very difficult to indicate, before qualification for PRRT, whether or not the patient will respond to the therapy and what the maximal tumor size change will be and whether PRRT may be considered as a neoadjuvant therapy. Moreover, in our work, the reduction of tumor volume was significant in many cases, but in two cases, we observed disease progression which means that PRRT did not always bring about the expected outcome. Finally, we also counted the percentage of tumor size shrinkage after PPRT, and we found that the response to therapy in both groups (pNETs vs. non-pNETs) was similar. Unfortunately, the cardinality of the group studied in our work was too small to draw unequivocal conclusions as to whether the use of different PRRT types and schemes brings about the same results. Among many radiological and clinical features, only negative results of $[18 \mathrm{~F}] \mathrm{FDG} \mathrm{PET} / \mathrm{CT}$ examination, histopathological grading (23), and to some extent the good SSTR expression on SRI (24) are widely known indicators of prognosis in NET patients. Nevertheless, none of those parameters are confirmed as factors influencing PRRT to be a neoadjuvant therapy.

It should also be considered that the use of different radionuclides (Y-90 and Lu-177 or mixed Y-90/Lu-177 having different radiation lengths and energies) may have an impact on the final outcome of the treatment. However, there are currently no studies comparing the different types of radionuclides used for PRRT.

Although we have not found an association between clinical and radiological features which could be helpful in proper patient selection for neoadjuvant PRRT, it is worth noting the possibility of the multigenomic blood mRNA biomarker (NETest) and PRRT predictive quotient (PPQ) use. PPQ had been evaluated as a predictor of PRRT response in 97\%. NETest accurately monitors PRRT response and is an effective surrogate marker of PRRT radiological response (25). Perhaps, it will be possible to use those parameters, facilitating the selection of patients who have a greater chance for radical surgery after neoadjuvant therapy.

Despite the lack of serious adverse events in our cohort, PRRT may be associated with the risk of short- and long-term side effects. Most side effects are connected directly with myelosuppression reversible and rather dose-limiting, but the problem of long-term complications remains crucial due to the expected long-time survival in radically treated patients. The most important long-term complications include myelodysplastic syndrome, acute myeloid leukemia, or bone marrow aplasia with the median latency period at diagnosis about 41 months (26). The prevalence of those severe, delayed adverse hematological events is estimated at $1.4 \%-4 \%$ (27, 28). In case of PRRT radiopharmaceuticals labeled with 90Y, kidney-
A

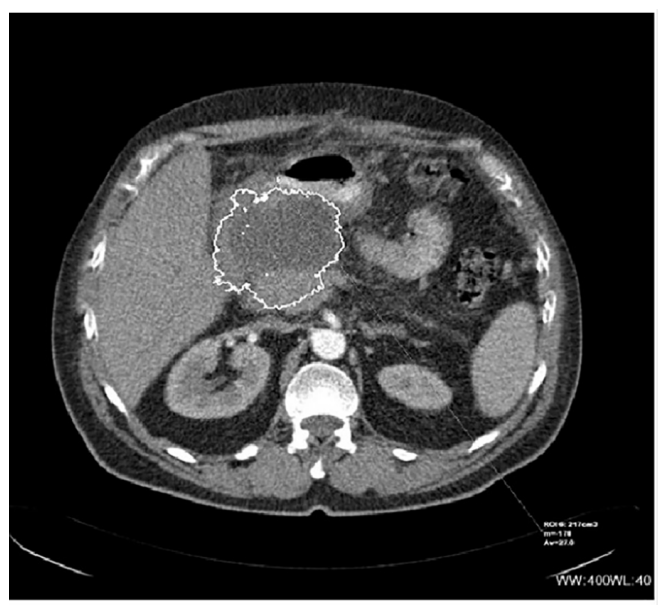

B

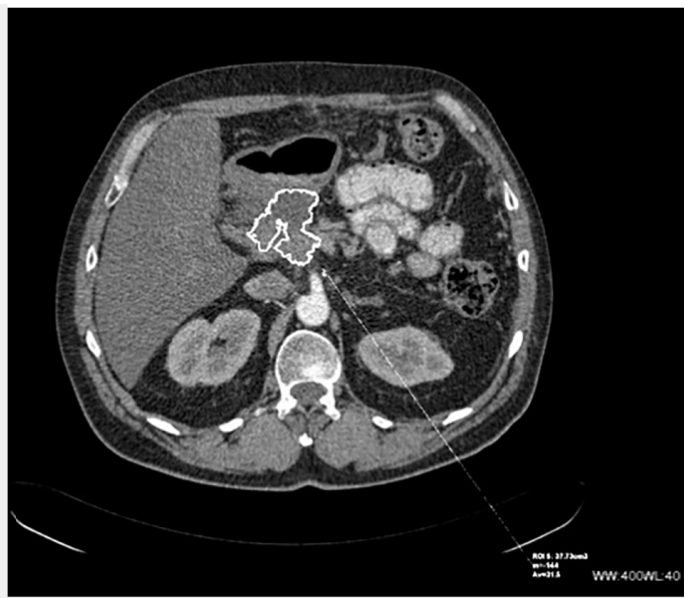

FIGURE 1 | CT scans of inoperable (before and after PRRT) NET of the pancreas: (A) before PRRT and (B) 4 months after 4 cycles of 90Y-DOTA-TATE. 

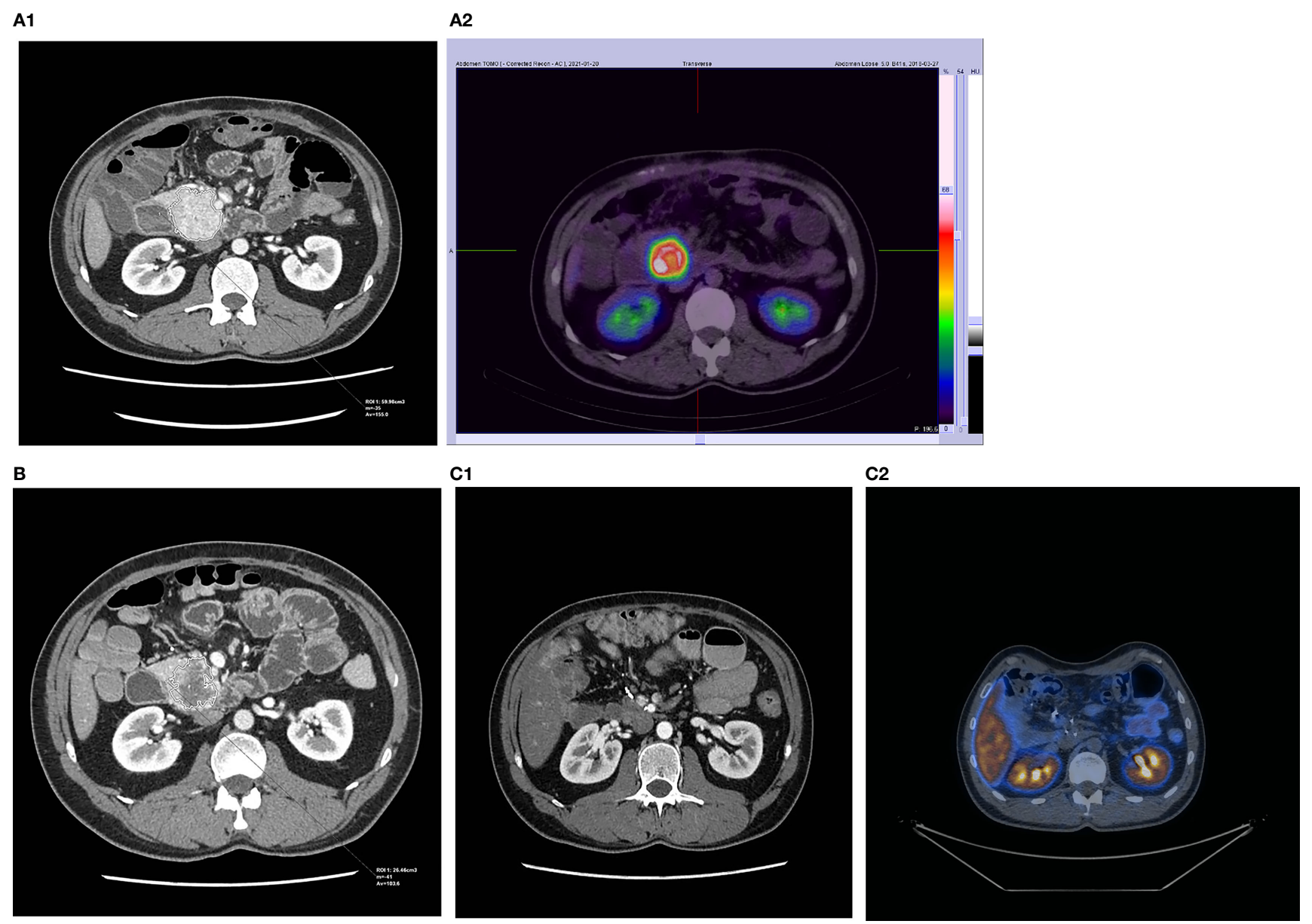

FIGURE 2 | CT and SRI scans of a patient with successfully operated NET of the pancreas: (A1, 2) before PRRT, (B) 3 months after 4 cycles of 177Lu/90Y-DOTATATE (only CT), and $(\mathbf{C 1}, \mathbf{2})$ after complete tumor removal.

related toxicity should also be considered (29). Some of those toxicities may be limited by proper dosimetry.

\section{CONCLUSIONS}

- In some cases of SSTR-positive NETs, PRRT used as a firstline treatment may cause significant tumor size reduction, which enables radical surgical intervention. In other cases (in majority of the patients), the benefits include reduction of total tumor burden and long-term stabilization of the disease according to RECIST criteria.

- To date, there are no clinical or radiological features (except high tumor burden) that give a fully unambiguous answer to the question of whether PRRT may allow for radical surgical treatment.

- All PRRT regimens can be considered as a useful therapy for somatostatin receptor-positive NETs, including the application of PRRT as a neoadjuvant therapy in primary inoperable tumors. Currently, there are no data indicating which PRRT regimen (177Lu, 90Y/177Lu, 90Y; TATE/TOC) and schemes could be most effective.

- PRRT was clinically well tolerated and did not interfere with the subsequent surgical or oncological treatment.

\section{DATA AVAILABILITY STATEMENT}

The original contributions presented in the study are included in the article/supplementary material. Further inquiries can be directed to the corresponding author.

\section{ETHICS STATEMENT}

The study protocol was approved by the Local Ethics Committee of the Jagiellonian University in Krakow (reference number: 1072.6120.32.2021). The patients/participants provided their written informed consent to participate in this study. 


\section{AUTHOR CONTRIBUTIONS}

MO: data collection, imaging review, data analysis, manuscript drafting, manuscript editing and approval, and study coordination. AS-S: data collection, imaging review, data analysis, manuscript drafting, and manuscript editing and

\section{REFERENCES}

1. Falconi M, Eriksson B, Kaltsas G, Bartsch DK, Capdevila J, Caplin M, et al. ENETS Consensus Guidelines Update for the Management of Patients With Functional Pancreatic Neuroendocrine Tumors and Non-Functional Pancreatic Neuroendocrine Tumors. Neuroendocrinology (2016) 103 (2):153-71. doi: 10.1159/000443171

2. Garcia-Carbonero R, Rinke A, Valle JW, Fazio N, Caplin M, Gorbounova V, et al. ENETS Consensus Guidelines for the Standards of Care in Neuroendocrine Neoplasms. Systemic Therapy 2: Chemotherapy. Neuroendocrinology (2017) 105(3):281-94. doi: 10.1159/000473892

3. Strosberg J, El-Haddad G, Wolin E, Hendifar A, Yao J, Chasen B, et al. Phase 3 Trial of (177)Lu-Dotatate for Midgut Neuroendocrine Tumors. NETTER-1 Trial Investigators. N Engl J Med (2017) 376(2):125-35. doi: 10.1056/ NEJMoa1607427

4. Del Prete M, Buteau FA, Arsenault F, Saighi N, Bouchard LO, Beaulieu A, et al. Personalized (177)Lu-Octreotate Peptide Receptor Radionuclide Therapy of Neuroendocrine Tumours: Initial Results From the P-PRRT Trial. Eur J Nucl Med Mol Imaging (2019) 46(3):728-42. doi: 10.1007/s00259-018-4209-7

5. Bodei L, Kidd M, Paganelli G, Grana CM, Drozdov I, Cremonesi M, et al. Long-Term Tolerability of PRRT in 807 Patients With Neuroendocrine Tumours: The Value and Limitations of Clinical Factors. Eur J Nucl Med Mol Imaging (2015) 42(1):5-19. doi: 10.1007/s00259-014-2893-5

6. Partelli S, Bertani E, Bartolomei M, Perali C, Muffatti F, Grana CM, et al. Peptide Receptor Radionuclide Therapy as Neoadjuvant Therapy for Resectable or Potentially Resectable Pancreatic Neuroendocrine Neoplasms. Surgery (2018) 163(4):761-7. doi: 10.1016/j.surg.2017.11.007

7. Parghane RV, Bhandare M, Chaudhari V, Ostwal V, Ramaswamy A, Talole S, et al. Surgical Feasibility, Determinants and Overall Efficacy Assessment of Neoadjuvant PRRT With 177Lu-DOTATATE for Locally Advanced Unresectable Gastroenteropancreatic Neuroendocrine Tumors. J Nucl Med (2021) 62(11):1558-63. doi: 10.2967/jnumed.120.258772

8. Fumagalli AA, Pellicciari MB, Reni AA, Borghesani MA, Zaninotto EA, Benini LA, et al. Role of Neoadjuvant PRRT in Resectable/Borderline Resectable PanNETs: Clinical, Pathological and Genomic Features. In: 18th Annual ENETS Conference for the Diagnosis and Treatment of Neuroendocrine Tumor Disease 25 - 27 February 2021 Booklet. (Virtual Conference, 2021). J Neuroendocrinol (2021) 33(Suppl 1):187-7. doi: 10.1111/jne.12950

9. Wang LF, Lin L, Wang MJ, Li Y. The Therapeutic Efficacy of $177 \mathrm{Lu}-$ DOTATATE/DOTATOC in Advanced Neuroendocrine Tumors: A MetaAnalysis. Medicine (Baltimore) (2020) 99(10):e19304. doi: 10.1097/ MD.0000000000019304

10. Saravana-Bawan B, Bajwa A, Paterson J, McEwan AJB, McMullen TPW. Efficacy of 177Lu Peptide Receptor Radionuclide Therapy for the Treatment of Neuroendocrine Tumors: A Meta-Analysis. Clin Nucl Med (2019) 44 (9):719-27. doi: 10.1097/RLU.0000000000002646

11. Kunz PL, Catalano PJ, Nimeiri H, Fisher GA, Longacre TA, Suarez CJ, et al. A Randomized Study of Temozolomide or Temozolomide and Capecitabine in Patients With Advanced Pancreatic Neuroendocrine Tumors: A Trial of the ECOG-ACRIN Cancer Research Group (E2211) [Abstract 4004]. J Clin Oncol (2018) 36(15_suppl):36. doi: 10.1200/JCO.2018.36.15_suppl.4004

12. Strosberg JR, Fine RL, Choi J, Nasir A, Coppola D, Chen DT, et al. First-Line Chemotherapy With Capecitabine and Temozolomide in Patients With Metastatic Pancreatic Endocrine Carcinomas. Cancer (2011) 117(2):268-75. doi: $10.1002 /$ cncr.25425

13. Xie H, Liu J, Yadav S, Keutgen XM, Hobday TJ, Strosberg JR, et al. The Role of Perioperative Systemic Therapy in Localized Pancreatic Neuroendocrine Neoplasms. Neuroendocrinology (2020) 110(3-4):234-45. doi: 10.1159/000501126 approval. AG: data collection, imaging review, manuscript drafting, and manuscript editing and approval. HO: data collection and manuscript editing and approval. AH-D: data collection, imaging review, data analysis, manuscript drafting, and manuscript editing and approval. All authors contributed to the article and approved the submitted version.

14. Ambe CM, Nguyen P, Centeno BA, Choi J, Strosberg J, Kvols L, et al. Multimodality Management of "Borderline Resectable" Pancreatic Neuroendocrine Tumors: Report of a Single-Institution Experience. Cancer Control (2017) 24(5):1073274817729076. doi: 10.1177/1073274817729076

15. van Vliet EI, van Eijck CH, de Krijger RR, Nieveen van Dijkum EJ, Teunissen JJ, Kam BL, et al. Neoadjuvant Treatment of Nonfunctioning Pancreatic Neuroendocrine Tumors With [177Lu-DOTA0,Tyr3] Octreotate. J Nucl Med (2015) 56(11):1647-53. doi: 10.2967/jnumed.115.158899

16. Schmidt MC, Uhrhan K, Fischer T, Schmitz S, Markiefka B, Drzezga A, et al. Complete Remission of Metastatic Neuroendocrine Paragastric Carcinoma After "Neoadjuvant" Peptide Receptor Radionuclide Therapy and Surgery. Clin Nucl Med (2015) 40(8):667-9. doi: 10.1097/RLU.0000000000000805

17. da Silva TN, van Velthuysen MLF, van Eijck CHJ, Teunissen JJ, Hofland J, de Herder WW. Successful Neoadjuvant Peptide Receptor Radionuclide Therapy for an Inoperable Pancreatic Neuroendocrine Tumour. Endocrinol Diabetes Metab Case Rep (2018) 2018:18-0015. doi: 10.1530/EDM-18-0015

18. Stoeltzing O, Loss M, Huber E, Gross V, Eilles C, Mueller-Brand J, et al. Staged Surgery With Neoadjuvant 90Y-DOTATOC Therapy for Down-Sizing Synchronous Bilobular Hepatic Metastases From a Neuroendocrine Pancreatic Tumor. Langenbecks Arch Surg (2010) 395(2):185-92. doi: 10.1007/s00423-009-0520-x

19. Ezziddin S, Lauschke H, Schaefers M, Meyer C, van Essen M, Biersack HJ, et al. Neoadjuvant Downsizing by Internal Radiation: A Case for Preoperative Peptide Receptor Radionuclide Therapy in Patients With Pancreatic Neuroendocrine Tumors. Clin Nucl Med (2012) 37(1):102-4. doi: 10.1097/ RLU.0b013e318238f111

20. Hirmas N, Jadaan R, Al-Ibraheem A. Peptide Receptor Radionuclide Therapy and the Treatment of Gastroentero-Pancreatic Neuroendocrine Tumors: Current Findings and Future Perspectives. Nucl Med Mol Imaging (2018) 52(3):190-9. doi: 10.1007/s13139-018-0517-x

21. Satapathy S, Mittal BR. 177Lu-DOTATATE Peptide Receptor Radionuclide Therapy Versus Everolimus in Advanced Pancreatic Neuroendocrine Tumors: A Systematic Review and Meta-Analysis. Nucl Med Commun (2019) 40(12):1195-203. doi: 10.1097/MNM.0000000000001103

22. Sowa-Staszczak A, Chrzan R, Pach D, Stefańska A, Tomaszuk M, BuziakBereza M, et al. Are RECIST Criteria Sufficient to Assess Response to Therapy in Neuroendocrine Tumors? Clin Imaging (2012) 36(4):360-4. doi: 10.1016/ j.clinimag.2011.11.005

23. Albertelli M, Dotto A, Di Dato C, Malandrino P, Modica R, Versari A, et al. PRRT: Identikit of the Perfect Patient. Rev Endocr Metab Disord (2021) 22 (3):563-79. doi: 10.1007/s11154-020-09581-6

24. Werner RA, Lapa C, Ilhan H, Higuchi T, Buck AK, Lehner S, et al. Survival Prediction in Patients Undergoing Radionuclide Therapy Based on Intratumoral Somatostatin-Receptor Heterogeneity. Oncotarget (2017) 8 (4):7039-49. doi: 10.18632/oncotarget.12402

25. Bodei L, Kidd MS, Singh A, van der Zwan WA, Severi S, Drozdov IA, et al. PRRT Neuroendocrine Tumor Response Monitored Using Circulating Transcript Analysis: The NETest. Eur J Nucl Med Mol Imaging (2020) 47 (4):895-906. doi: 10.1007/s00259-019-04601-3

26. Bergsma H, van Lom K, Raaijmakers MHGP, Konijnenberg M, Kam BLBLR, Teunissen JJM, et al. Persistent Hematologic Dysfunction After Peptide Receptor Radionuclide Therapy With ${ }^{177}$ Lu-DOTATATE: Incidence, Course, and Predicting Factors in Patients With Gastroenteropancreatic Neuroendocrine Tumors. J Nucl Med (2018) 59(3):452-8. doi: 10.2967/ jnumed.117.189712

27. Chantadisai M, Kulkarni HR, Baum RP. Therapy-Related Myeloid Neoplasm After Peptide Receptor Radionuclide Therapy (PRRT) in 1631 Patients From Our 20 Years of Experiences: Prognostic Parameters and Overall Survival. Eur 
J Nucl Med Mol Imaging (2021) 48(5):1390-8. doi: 10.1007/s00259-02005127-9

28. Sabet A, Ezziddin K, Pape UF, Ahmadzadehfar H, Mayer K, Pöppel T, et al. Long-Term Hematotoxicity After Peptide Receptor Radionuclide Therapy With 177Lu-Octreotate. J Nucl Med (2013) 54(11):1857-61. doi: 10.2967/ jnumed.112.119347

29. Valkema R, Pauwels SA, Kvols LK, Kwekkeboom DJ, Jamar F, de Jong M, et al. Long-Term Follow-Up of Renal Function After Peptide Receptor Radiation Therapy With (90)Y-DOTA(0),Tyr(3)-Octreotide and (177)Lu-DOTA(0), Tyr(3)-Octreotate. J Nucl Med (2005) 46(Suppl 1):83S-91S.

Conflict of Interest: The authors declare that the research was conducted in the absence of any commercial or financial relationships that could be construed as a potential conflict of interest.
Publisher's Note: All claims expressed in this article are solely those of the authors and do not necessarily represent those of their affiliated organizations, or those of the publisher, the editors and the reviewers. Any product that may be evaluated in this article, or claim that may be made by its manufacturer, is not guaranteed or endorsed by the publisher.

Copyright (c) 2021 Opalińska, Sowa-Staszczak, Grochowska, Olearska and Hubalewska-Dydejczyk. This is an open-access article distributed under the terms of the Creative Commons Attribution License (CC BY). The use, distribution or reproduction in other forums is permitted, provided the original author(s) and the copyright owner(s) are credited and that the original publication in this journal is cited, in accordance with accepted academic practice. No use, distribution or reproduction is permitted which does not comply with these terms. 